\title{
STATUS OF THE IMAGING SPECTROSCOPY MISSION ENMAP WITH RADIOMETRIC CALIBRATION AND CORRECTION
}

\author{
T. Storch ${ }^{1, *}$, H.-P. Honold ${ }^{2}$, K. Alonso ${ }^{1}$, M. Pato ${ }^{1}$, M. Mücke ${ }^{2}$, P. Basili ${ }^{2}$, S. Chabrillat ${ }^{3}$, S. Fischer ${ }^{4}$ \\ ${ }^{1}$ Earth Observation Center (EOC), German Aerospace Center (DLR), Germany - tobias.storch@dlr.de \\ ${ }^{2}$ OHB System AG, Germany \\ ${ }^{3}$ Helmholtz Center Potsdam, German Research Center for Geosciences (GFZ), Germany \\ ${ }^{4}$ Space Administration, German Aerospace Center (DLR), Germany
}

Commission I, WG I/1

KEY WORDS: Imaging Spectroscopy, EnMAP, Remote Sensing Satellite, Radiometric Calibration, Radiometric Correction

\begin{abstract}
:
The high-resolution imaging spectroscopy remote sensing mission EnMAP (Environmental Mapping and Analysis Program, enmap.org) covers the spectral range from $420 \mathrm{~nm}$ to $2450 \mathrm{~nm}$ with a spectral sampling distance varying between $4.8 \mathrm{~nm}$ and $12.0 \mathrm{~nm}$ comprising 262 spectral bands. We focus on the planned framework concerning radiometry. The expected signal-to-noise ratio at reference radiance level is 500:1 at $495 \mathrm{~nm}$ and 150:1 at $2200 \mathrm{~nm}$. The radiometric resolution is 14 bits and an absolute radiometric accuracy of better than $5 \%$ is achieved. Radiometric calibration is based on Sun calibration measurements with a fullaperture diffusor for absolute calibration. In addition, relative calibration monitors the instrument during the complete mission lifetime based on an integrating sphere (on the satellite). The fully-automatic on-ground image processing chain considers the derived radiometric calibration coefficients in the radiometric correction which is followed by the orthorectification and atmospheric compensation. Each of the two 2-dimensional detector arrays of the prism-based pushbroom dual-spectrometer works in a dual-gain configuration to cover the complete dynamic range. EnMAP will acquire $30 \mathrm{~km}$ in the across-track direction with a ground sampling distance of $30 \mathrm{~m}$ and the across-track tilt capability of $30^{\circ}$ will enable a target revisit time of less than 4 days. The launch is scheduled for 2021. The high-quality products will be freely available to international scientific users for measuring and analysing diagnostic parameters which describe vital processes on the Earth's surface.
\end{abstract}

\section{INTRODUCTION}

Most activities in imaging spectroscopy in the last decades have been based on airborne imaging spectrometers covering the visible and near-infrared (VNIR, roughly $400 \mathrm{~nm}$ to $1000 \mathrm{~nm}$ ) and shortwave-infrared (SWIR, roughly $1000 \mathrm{~nm}$ to $2500 \mathrm{~nm}$ ) reflective spectral ranges. Especially, the Airborne Visible/Infrared Imaging Spectrometer (AVIRIS), realized and operated by the NASA Jet Propulsion Laboratory in California, has been used in a large number of imaging spectroscopy campaigns for the last decades. Unfortunately, the potential of imaging spectroscopy is not counterbalanced by an equivalent availability of spaceborne imaging spectrometers. In particular, the technology demonstration mission Hyperion on Earth Observing 1 (EO-1), realized and operated by NASA and USGS, has been the main provider of satellite hyperspectral data for the last years. Recently, DESIS and PRISMA were launched in 2018 and 2019 as well as HISUI and EMIT will be launched in 2020 and 2021. Furthermore, imaging spectroscopy mapping missions from ESA with CHIME and NASA with SBG are planned for later than 2025 .

\section{MISSION}

The imaging spectroscopy remote sensing mission EnMAP (Environmental Mapping and Analysis Program, enmap.org) will significantly contribute to the availability of space-based imaging spectroscopy products (Guanter et al., 2015).
The mission relies on a prism-based dual-spectrometer (Kaufmann et al., 2016). The VNIR spectrometer covers the spectral range from $420 \mathrm{~nm}$ to $1000 \mathrm{~nm}$ with a spectral sampling distance between $4.8 \mathrm{~nm}$ and $8.2 \mathrm{~nm}$. The SWIR spectrometer covers the spectral range from $900 \mathrm{~nm}$ to $2450 \mathrm{~nm}$ with a spectral sampling distance between $7.4 \mathrm{~nm}$ and $12.0 \mathrm{~nm}$. Thus, the total number of spectral bands is 262 and for both instruments the spectral resolution is about a factor of 1.2 larger than the spectral sampling distance. We focus on the planned framework concerning radiometry. A signal-to-noise ratio at reference radiance level (which is defined by 30\% surface albedo, $30^{\circ}$ Sun zenith angle, ground at sea level, and $40 \mathrm{~km}$ visibility with rural atmosphere) of 500:1 at $495 \mathrm{~nm}$ and 150:1 at $2200 \mathrm{~nm}$ is achieved, where the radiometric resolution is 14 bits. For both detectors, the signal is fed to two parallel amplifiers with different gains. For VNIR an automatic gain switching and for SWIR a fixed gain setting is applied. In-flight radiometric calibration is based on Sun calibration measurements with a full-aperture diffusor for absolute calibration. In addition, a frequent relative calibration monitors the instrument during the complete mission lifetime based on an integrating sphere (on the satellite) coated with Spectralon and illuminated with a Tungsten halogen lamp and a white LED (Light Emitting Diode). These calibration measurements also allow for the regular update of the dead pixel mask containing e.g. hot and cold pixels. The frequency of the absolute calibration is scheduled based on a model using previous calibration measurements to minimize the aging of the diffusor.

* Corresponding author 
The calibration is complemented with closed shutter measurements before and after each observation for dark signal subtraction and additional deep space looking measurements for shutter thermal emission monitoring. Due to air-vacuum transition and gravity release, most significant effects are expected during and shortly after launch. Therefore, a high frequency of calibration measurements is planned during the commissioning phase. Aging effects within the operational phase can be studied with longer calibration intervals. Pre-flight radiometric calibration also considers effects which are not measureable during operations e.g. spectral and spatial straylight, and verify temperature and radiometric stability assumptions. Furthermore, this provides the first reference measurements of the calibration equipment on the satellite. Further calibration equipment (on the satellite), especially a doped sphere with spectral features, enables a spectral accuracy of better than $0.5 \mathrm{~nm}$ in VNIR and $1.0 \mathrm{~nm}$ in SWIR. Each detector array has 1000 valid pixels in spatial direction with an instantaneous field-of-view of 9.5 arcsec. Thereby, a geometric resolution of $30 \mathrm{~m} \times 30 \mathrm{~m}$ and a swath width (across-track) of $30 \mathrm{~km}$ is realized, because the orbit with a repeat cycle of 398 revolutions in 27 days has an altitude of $652 \mathrm{~km}$. A swath length (along-track) of $5000 \mathrm{~km}$ can be acquired per day and an across-track tilt capability of $30^{\circ}$ enables a target revisit time of less than 4 days. The local time of descending node is 11:00 hrs $\pm 17 \mathrm{~min}$. It is remarked that the two 2-dimensional detector arrays are not spatially aligned, namely there is a shift of approximately 190 arcsec along track which corresponds to approximately $600 \mathrm{~m}$ on ground. A geometric accuracy of 100 $\mathrm{m}$ is achieved - improved by on-ground processing to $30 \mathrm{~m}$ with respect to a used reference image (Schneider et al., 2012).

The launch is scheduled for 2021 with an operational lifetime of 5 years. The mission is in its testing phase. The hyperspectral image products at different processing levels will be freely available to the scientific user community for measuring and analysing bio-, geochemical, and physical parameters characterizing the Earth's surface on a global scale. This encompasses agriculture, forestry, soil and geological environments, as well as coastal zones and inland waters.

The OHB System AG is responsible for realizing the space segment and the Earth Observation Center (EOC), together with the German Space Operations Center (GSOC), of the German Aerospace Center (DLR) is responsible for establishing and operating the ground segment (Storch et al., 2013). The German Research Center for Geosciences (GFZ) is responsible for the science segment and the DLR Space Administration covers the mission management.

We give an overview of the status of EnMAP in Section 3, detail the radiometric aspects with the calibration in Section 4, the correction in Section 5, and conclude in Section 6.

\section{STATUS}

Both the space and ground segment are in the final stages of the production and already perform verification activities. The space segment has characterized major elements and performed some environmental tests successfully. The pre-flight characterization and calibration campaign of the instrument is scheduled for mid of 2020 . The ground segment tested its major interfaces, the image processing chain is realized, and also the web-interface to the users for ordering observations and products is in technical verification. The planning for the operational validation is scheduled for mid of 2020. The science segment is active since many years to support the mission at present focussing on algorithm consolidation and education initiatives with airborne campaigns for the user community.

\section{RADIOMETRIC CALIBRATION}

The in-flight radiometric calibration is based on frequent relative calibrations for monitoring the instrument as discussed in Section 4.2 and the absolute calibrations for generating the radiometric calibration coefficients during the complete mission lifetime is discussed in Section 4.3. All calibration and Earth observations are accomplished by closed shutter measurements, which are specified in Section 4.1. These activities are complemented by comprehensive pre-flight radiometric characterizations also of the calibration equipment on the satellite (as illustrated in Figure 1) to verify the behaviour of the instrument and to generate first radiometric reference and calibration tables. Most of these parameters are re-determined in-flight, but others cannot be retrieved or can be retrieved at insufficient accuracy only. With relevance to radiometry these are in particular: response non-uniformity, spectral response function and bandwidth including temperature dependencies, polarization sensitivity, straylight considerations as well as the Bidirectional Reflectance Distribution Function (BRDF) of the full-aperture diffusor for Sun calibration. The reference tables refer to values averaged over time for each detector element for the various operational calibration modes of the instrument. The data is used for an evaluation of calibration measurements during operations as well as to monitor changes in the instrument behaviour. The idea behind this approach is that, as long as the instrument characteristics change by small amounts, the corresponding measurements are reproducible with high accuracy. Whenever larger deviations between a measurement and the reference occur, a more detailed analysis along with a measurement repetition is carried out. This approach helps to exclude punctual measurement anomalies from actual instrument changes. Most significant changes are expected during and shortly after launch due to air-vacuum transition and gravity release. Therefore, a high frequency of calibration measurements is planned during the commissioning phase (Hollstein et al., 2015). Because the radiometric calibration depends on the spectral calibration, we assume that the spectral calibration is correctly performed and otherwise spectral and radiometric calibrations have to be carried out in an iterative manner (Storch et al., 2018).

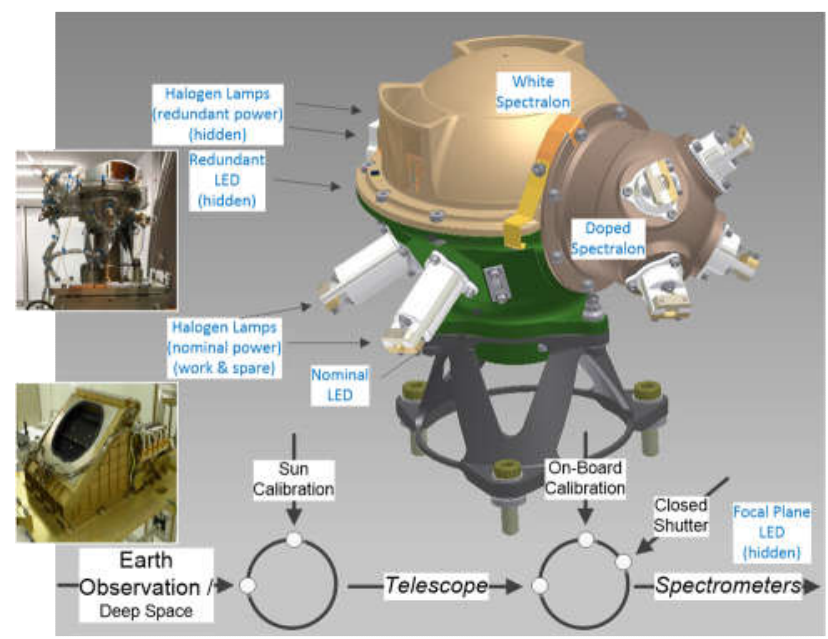

Figure 1. Calibration equipment on the satellite. (Image by OHB System AG.) 


\subsection{Closed Shutter Measurement}

Every calibration and Earth observation is accompanied by at least two series of closed shutter measurements for all spectral and spatial pixels to correct the dark signal of the VNIR and SWIR detectors. They are the most frequent measurements to characterize the instrument. Typically, series consist of measurements in low gain and high gain (128 frames each) and the first series starts before and the second after the observation measurements. Processing and analysis of the signals consists of: checking for outliers, flagging of dead pixels, averaging the measurements separately for the two gains and series, comparing the measurements to the references, and perform an interpolation between the series, if necessary.
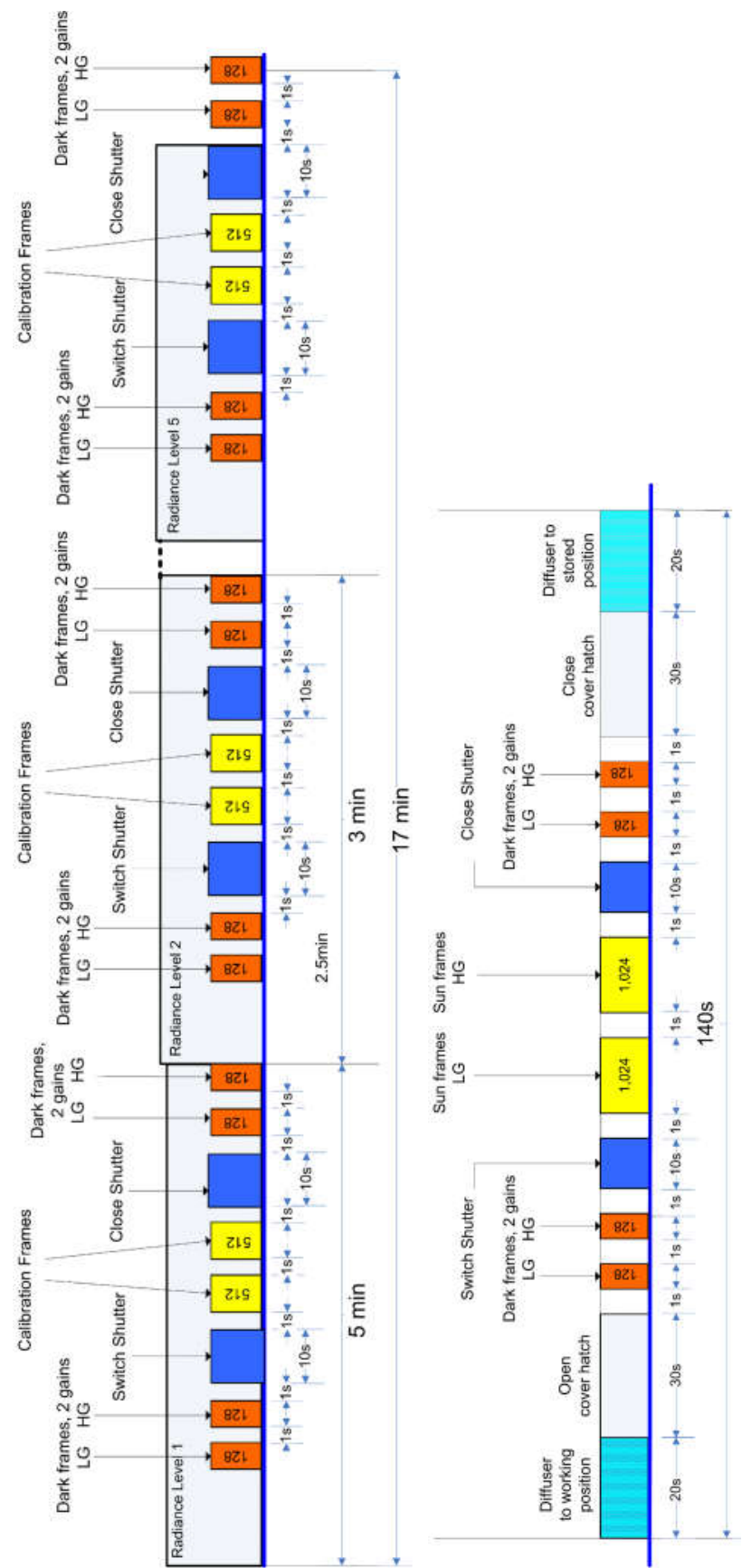

Figure 2. Sequence for relative (left) and absolute (right) radiometric calibration. (Images by OHB System AG.)
An additional step is performed for the SWIR detector. Unlike in VNIR, the shutter causes a residual radiation in the SWIR which leads to an error in the measurement of the dark signal. This effect, which is expected to be minor, is corrected by using values obtained by deep space looking measurements (1024 frames for each of the two gains) since the shutter temperature is expected to be constant. Therefore, these calibrations are expected to be performed every quarter. The signal caused by radiation from the shutter is calculated as difference between the signal measured at closed shutter and that measured at open shutter for deep space looking.

\subsection{Relative Calibration}

Relative radiometric calibration is based on measurements of an integrating sphere (on the satellite) coated with Spectralon and illuminated with a Tungsten halogen lamp and a white LED. They are operated at five different currents and illuminate the entire entrance slit. These lights are configured redundantly to obtain a high reliability. However, knowledge of aging of the
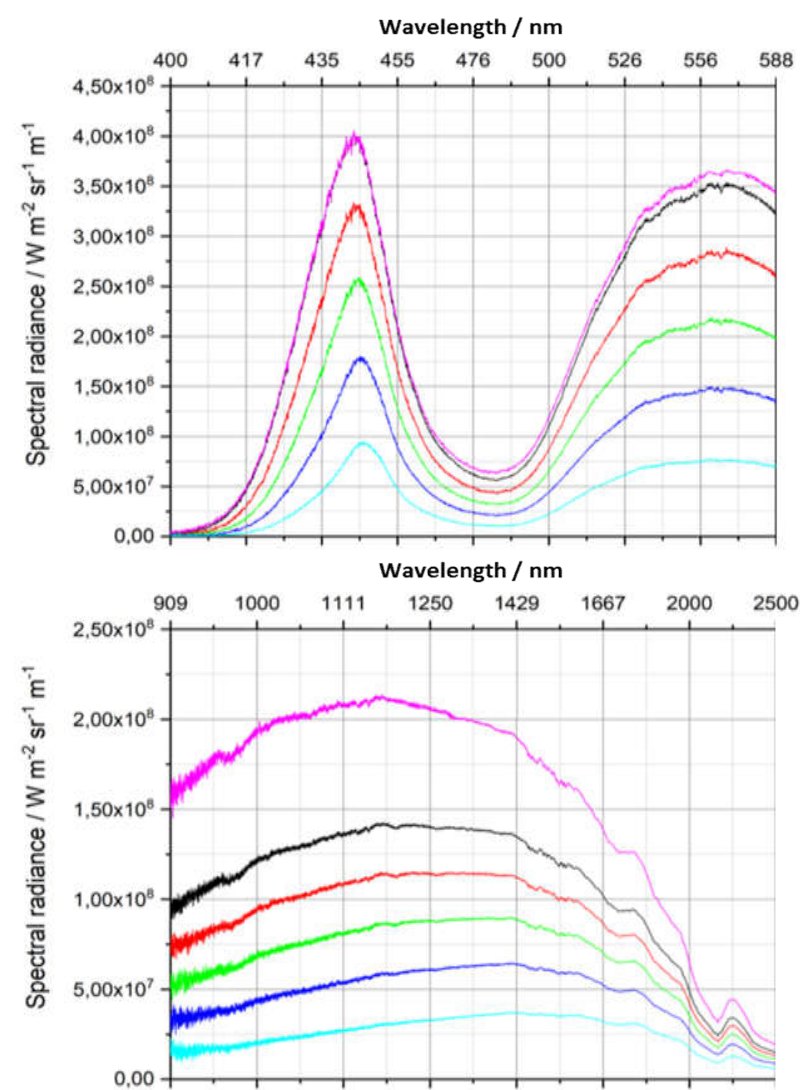

\begin{tabular}{|l|l|l|}
\hline Illumination Level & $\begin{array}{l}\text { Current Tungsten } \\
\text { halogen lamp }\end{array}$ & $\begin{array}{l}\text { Current white } \\
\text { LED }\end{array}$ \\
\hline IL1 $(60 \% / 90 \%)$ & $716.7 \mathrm{~mA}$ & $855.6 \mathrm{~mA}$ \\
\hline IL2 $(48 \% / 72 \%)$ & $674.5 \mathrm{~mA}$ & $634.4 \mathrm{~mA}$ \\
\hline IL3(36\%/54\%) & $626.9 \mathrm{~mA}$ & $455.7 \mathrm{~mA}$ \\
\hline IL4 (24\%/36\%) & $596.3 \mathrm{~mA}$ & $282.0 \mathrm{~mA}$ \\
\hline IL5(12\%/18\%) & $491.6 \mathrm{~mA}$ & $134.5 \mathrm{~mA}$ \\
\hline IQ/IL1 $(90 \% / 90 \%)$ & $807.3 \mathrm{~mA}$ & $855.6 \mathrm{~mA}$ \\
\hline
\end{tabular}

Figure 3. Characterization of integrating sphere for part of VNIR (top) and SWIR (middle) at different illumination levels (bottom). (Image by PTB, OHB System AG.) 
lights and integrating sphere are reduced as well as different parts of the optics are covered compared to Earth observations. E.g. the telescope is not considered, but the shutter is considered. Therefore, these calibrations are applied for monitoring the radiometric stability and relative changes, but not for absolute radiometric calibration. These calibrations are expected to be performed at a high frequency of every week during the complete mission lifetime.

The sequence for a relative radiometric calibration is illustrated in Figure 2 (left).

The burn-in time of the lamps is accounted for in data read-out and dark signal measurements using the shutter are performed at the beginning and the end of each illumination level. For each illumination level the focal plane is operated in low gain as well as in high gain.

At the bottom of Figure 3 the specification of the different Illumination Levels (IL) with an additional IL at maximal currents is stated. The pre-flight characterization is illustrated at the top (for part of VNIR) and middle (for part of SWIR) of Figure 3 using a vacuum Fourier-transformation spectrometer with three beam-splitter detectors and two radiative transfer standards. To study the homogeneity five field positions $(0 \mathrm{~mm}$, $\pm 6 \mathrm{~mm}$, and $\pm 11.5 \mathrm{~mm}$ ) are considered for all four light sources (nominal and redundant with work and spare) and to study the stability three temperatures $\left(20^{\circ} \mathrm{C}, 21^{\circ} \mathrm{C}\right.$, and $\left.22^{\circ} \mathrm{C}\right)$ are considered. The measurements of Figure 3 also illustrate the resulting signal of the integrating sphere for the different IL and the closed shutter measurements between the different IL.

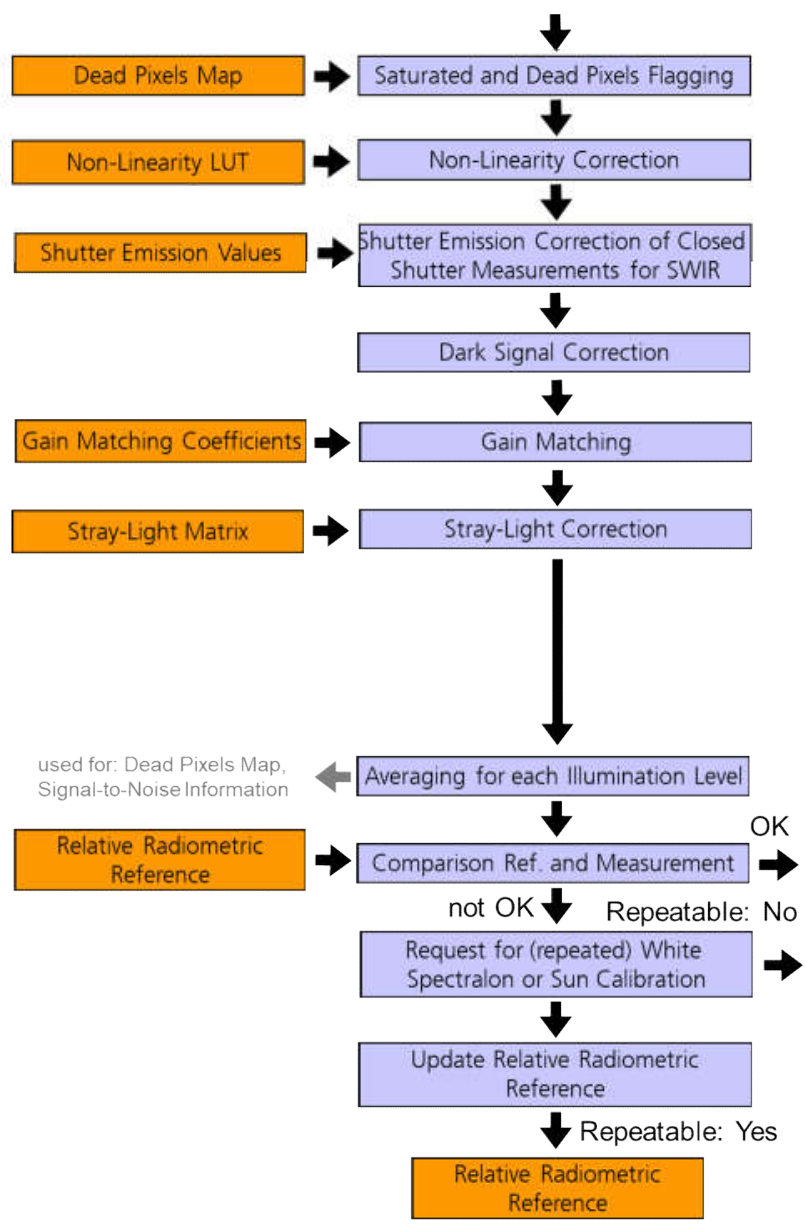

Figure 4. Corrections for relative radiometric calibrations.
The complete correction for relative radiometric calibrations is illustrated in Figure 4, where outliers are considered during averaging, with the potential update of the reference, if limits are violated.

\subsection{Absolute Calibration}

Absolute radiometric calibration is based on Sun calibration measurements with a full-aperture diffusor. Assuming a nominal behaviour of the instrument, a low frequency of every month is expected to be sufficient. However, based on the statistics of already performed Sun calibrations and the monitored performance of the instrument, an even lower frequency is possible. This further reduces the aging of the diffusor which is critical for the accuracy of the radiometric calibration. To perform the absolute calibration, which is carried out for low gain and high gain, the satellite is tilted such that the Sun entrance baffle points directly to the Sun. During the positioning of the satellite the Sun diffuser is moved into Sun calibration position and the cover hatch is opened. The Sun calibration is acquired at specific positions in orbit to minimize straylight from the Earth.

The sequence for an absolute radiometric calibration is illustrated in Figure 2 (right).

The complete correction for absolute radiometric calibrations is illustrated in Figure 5, where outliers are considered during averaging, with the potential update of the reference and calibration coefficients, if limits are violated.

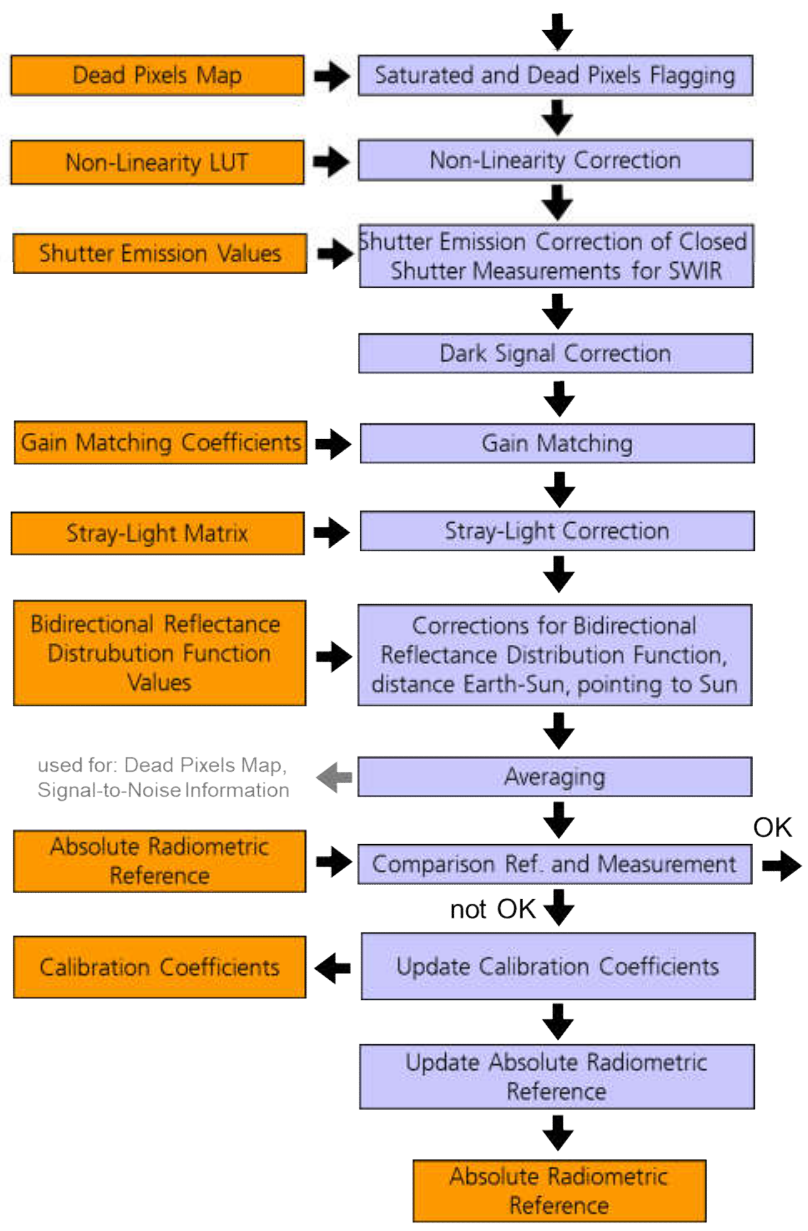

Figure 5. Corrections for absolute radiometric calibrations. 
If the measurements are out of limits compared to the reference, namely half of the required radiometric accuracy, detailed analysis is necessary to identify the reasons for the changes in the instrument performance; and the data are not solely archived for purposes of monitoring statistics. In case of significant changes, typically an additional Sun calibration is performed to confirm the results and to avoid erroneous conclusions. Furthermore, the results shall be confirmed by additional independent measurements such as relative radiometric calibrations. Some causes for deviations in Sun calibrations are: changes in instrument radiometry by degradations in optics or detectors, changes in instrument spectral characteristics, inaccurate Sun pointing or positioning of the full-aperture diffuser, challenges with the instrument health such as the focal planes or temperatures, or degradations of the full-aperture diffusor. Therefore, extensive analysis and additional measurements are required to identify the cause.

The radiometric calibration coefficients consist of one file for each sensor. Each file contains the gain matching coefficients, the radiometric coefficients for each band, and the response non-uniformity for each pixel. During Sun calibration the irradiance from the Sun is converted to radiance by means of the diffuser, where the extraterrestrial irradiance values of the Sun are considered as well as the BRDF of the full-aperture diffusor and the distance between Sun and satellite.

During operations the different types of Calibration Requests (CR) are submitted to the planning system by users responsible for calibration and the resulting CR observations are ingested to the sequence-of-events of the satellite with high priority.

\section{RADIOMETRIC CORRECTION}

Similar to CR, Earth observation requests are submitted to the planning system by the users, ingested to the sequence-ofevents of the satellite. Earth observation requests are specified by the centre coordinate, swath length, time window, off-nadir angle, sun glint, and cloud coverage. After receiving, archiving, and processing, as discussed in Section 5.1 in general and specifically concerning radiometry in Section 5.2, the products are delivered to the users.

\subsection{Image Processing Chain}

The processing chain, which is established on-ground, generates fully-automatic standardized high-quality products at different levels for the international scientific users (Müller et al, 2010).

The Level 0 processor generates time-tagged instrument raw data with auxiliary information which are long-term archived, but not distributed to the users. It performs unpacking, decompression, screening, dark signal extraction, and tiling to areas of about $30 \mathrm{~km} \times 30 \mathrm{~km}$. Afterwards, Level 1A/1B, 1C, and $2 \mathrm{~A}$ processing is performed to finally generate consistent metadata and quicklooks. This includes the establishment of ground control points using image matching techniques and a reference image database and digital elevation model to obtain a robust sensor model refinement for the complete acquisition. Operational data quality control, namely a quantitative assessment of various properties, is provided for each processing step to account for the demand in highly reliable, well documented and standardized data products. By this process, the valid functionality of the sensor and processing chain is investigated, and thus, ensured.

The Level 1A/1B processor generates at-sensor radiances as detailed in Section 5.2. Furthermore, the data are not resampled, spectrally and geometrically characterized, and annotated e.g. with pixel classification (usability mask) and information necessary for later processing.

The Level 1C processor orthorectifies Level 1B products to a user selected map projection (UTM, geographic, or European projection LAEA) using a user selected resampling method (nearest neighbour, bi-linear interpolation, or cubic convolution). Direct georeferencing is applied using the physical sensor model (with a correction of sensor interior orientation, satellite motion, light aberration and refraction) and terrain related distortions from imagery.

The Level $2 \mathrm{~A}$ processor atmospherically compensates Level 1C products to surface or underwater reflectances, namely different methods over land and water areas are applied including adjacency correction. Classifications (land-water-background, cloud, shadow, haze, cirrus, Sun glitter), aerosol optical thickness, and columnar water vapour are estimated.

Level $1 \mathrm{~A} / \mathrm{B}, 1 \mathrm{C}$, and $2 \mathrm{~A}$ products are generated to a user selected format (image data in BSQ, BIL, BIP, JPEG2000, or GeoTIFF and metadata in XML) and disseminated through web-based interfaces.

The catalogue allows users to search and browse all products based on the standardized protocols CSW (catalogue service for the web) and WMS (web mapping service). Because of the necessary various processing options, each product is specifically generated for each order and delivered using SFTP (secure file transfer protocol).

\subsection{Radiometric Correction Chain}

The complete radiometric correction chain for Earth observations is illustrated in Figure 6.

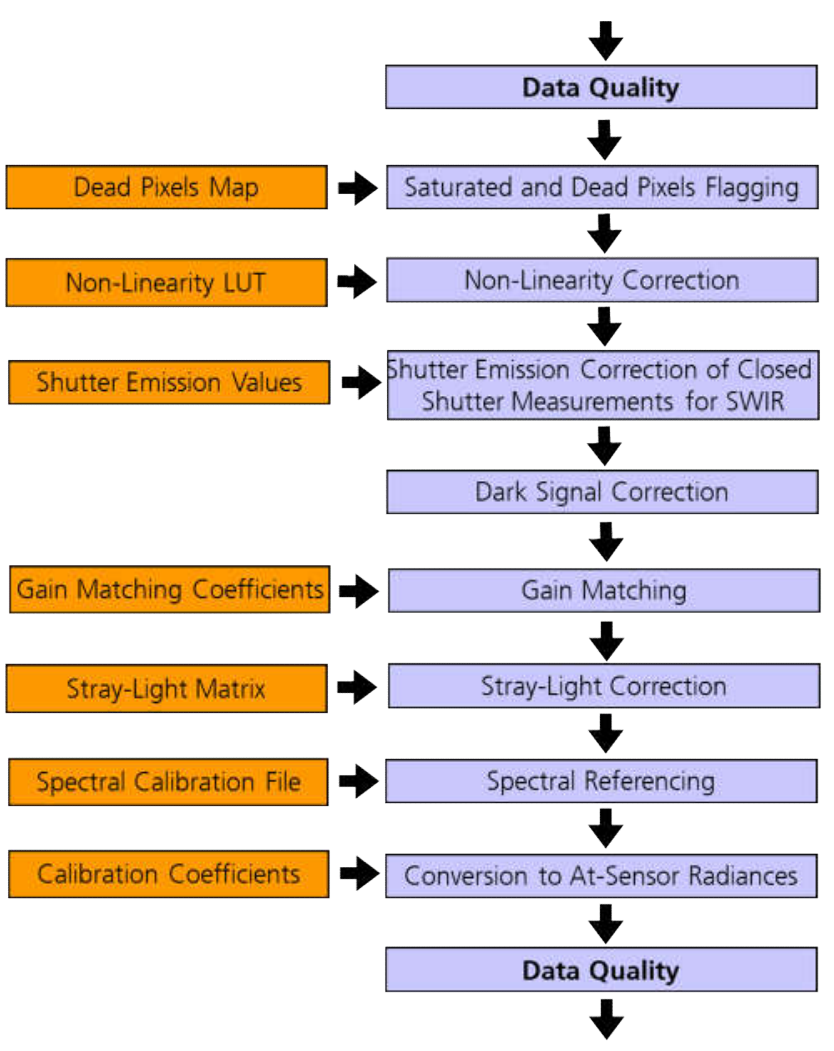

Figure 6. Radiometric correction chain. 
The data processing sequence starts with the flagging of dead pixels. This map of permanently corrupted pixels (no signal independent on illumination level (cold pixel) or signal appears permanently bright or saturated, even at low radiances such as for closed shutter measurements (hot pixel)) for each sensor is extracted from archive for the time of acquisition and the pixels are excluded from further processing.

Afterwards, the signal of the instrument is corrected for the non-linear response function of the pixels, which is especially important close to the borders of the sensitivity range. This is done for each pixel individually through non-linearity look-up tables, which depend on the sensor and gain. They are generated based on dedicated non-linearity calibration measurements using focal plane LED. The correction is applied to both closed shutter measurements and Earth observations.

The dark signal of the sensor is computed with the help of the closed shutter measurements before and after the imaging phase, which have already been corrected for non-linearity. The methods of filtering outliers, averaging, and considering the shutter thermal emission for SWIR for the closed shutter measurements are described in Section 4.1.

Next, it is necessary to match the trend of the pixels in low gain and high gain before performing straylight corrections, because the signal from any given pixel has contributions from neighboring spatial and spectral pixels, which may have different gains. This is accomplished applying a gain matching coefficient to pixels in low gain, where different tables are available for VNIR and SWIR.

Finally, the radiometric correction step consists of applying the response non-uniformity and radiometric calibration coefficients to each pixel. Similarly to gain matching, there are different tables for VNIR and SWIR.

The Level 1A/1B products contain the image for each sensor separately with 16-bit unsigned integer values of unit $\mathrm{W} / \mathrm{m}^{2} / \mathrm{sr} / \mathrm{nm}$. Also provided are quality flags generated by the data quality routines at the beginning and end of the radiometric correction chain. These quality flags enhance the information on the dead pixel map by identifying saturated pixels, pixels adjacent to saturated pixels to account for blooming effects, low-radiance pixels, and artefacts such as banding and striping. Based on this information an overall quality is aggregated for the complete image, but also separate measures are provided for VNIR and SWIR

This online data quality control for all products is complemented by offline data quality control for selected products.

\subsection{Verification}

A simulation chain enables the evaluation of optimal instrument configurations for radiometric, spectral, and geometric parameters as well as the evaluation and profiling of the onground processing chain (Segl et al., 2012).

The simulation of image acquisitions is based on high-spectral resolution airborne acquisitions with a small swath or lowspectral resolution spaceborne acquisitions with a large swath enhanced using spectral libraries. The instrument geometric, spectral, and radiometric parameters with the transformation from at-sensor radiance to photons, electrons, and finally to digital numbers are derived from analysis of the single effects and are specified by the corresponding manufacturer. The device imperfections and different noise sources, considering parameters such as integration time, quantum efficiency, optical transmittance, spectral sampling distance, detector element size, read, shot, dark, analog-to-digital conversions, and quantization noise, variable low gains and high gains, random dark signal,
SWIR infrared background signal, and non-linear detector responses for each detector element are taken into account. Based on these simulated products also the radiometric correction chain is verified pre-flight.

In-flight verification is performed especially during the commissioning phase by the space and ground segment, but additionally an independent validation of the radiance and reflectance products is carried out by the science segment. The overarching objective of the product validation activities is to evaluate the accuracies of the products independently of the data quality control activities. For all product levels, a set of parameters defining the quality is evaluated by means of ground-based and scene-based methods during all mission phases. The radiometric accuracies are assessed through specific field campaigns and through comparisons with automatic measurements from the RadCalNet network and with other optical missions having comparable spatial and spectral resolutions such as Sentinel-2. The radiometric stability is evaluated based on time series over pseudo-invariant calibration sites. Scene-based image processing methods is applied to check the signal-to-noise ratio.

\section{CONCLUSIONS}

We analysed the status of the imaging spectroscopy remote sensing mission EnMAP (Environmental Mapping and Analysis Program, enmap.org) with a focus on the activities planned concerning radiometric calibration and correction.

\section{ACKNOWLEDGEMENTS}

Supported by the DLR Space Administration with funds of the German Federal Ministry of Economic Affairs and Technology on the basis of a decision by the German Bundestag (50 EE 0850).

\section{REFERENCES}

Guanter, L., Kaufmann, H., Segl, K., Foerster, S., Rogass, C., Chabrillat, S., Kuester, T., Hollstein, A., Rossner, G., Chlebek, C., Straif, C., Fischer, S., Schrader, S., Storch, T., Heiden, U., Müller, A., Bachmann, M., Mühle, H., Müller, R., Habermeyer, M., Ohndorf, A., Hill, J., Buddenbaum, H., Hostert, P., van der Linden, S., Leitao, P.J., Rabe, A., Doerffer, R., Krasemann, H., Xi, H., Mauser, W., Hank, T., Locherer, M., Rast, M., Staenz, K., Sang, B., 2015. The EnMAP spaceborne imaging spectroscopy mission for Earth observation, Remote Sensing, 7(7), 8830-8857.

Hollstein, A., Rogass, C., Segl, K., Guanter, L., Bachmann, M., Storch, T., Müller, R., Krawczyk, H., 2015. EnMAP Radiometric Inflight Calibration, Post-Launch Product Validation, and Instrument Characterization Activities, In: Proc. IGARSS2015, Milan, Italy, 2806-2812.

Kaufmann, H., Sang, B., Storch, T., Segl, K., Foerster, S., Guanter, L., Erhard, M., Heider, B., Hofer, S., Honold, H.-P., Penné, B., Bachmann, M.; Habermeyer, M., Müller, A., Müller, R., Rast, M., Staenz, K., Straif, C., Chlebek, C., 2016. Environmental Mapping and Analysis Program - A German Hyperspectral Mission, In: Optical Payloads for Space Missions, John Wiley \& Sons, UK, 161-182. 
Müller, R., Bachmann, M., Makasy, C., de Miguel, A., Müller, A., Neumann, A., Palubinskas, G., Richter, R., Schneider, M., Storch, T., Walzel, T., Kaufmann, H., Guanter, L., Segl, K., Heege, T., Kiselev, V., 2010. The Processing Chain and $\mathrm{Cal} /$ Val Operations of the Future Hyperspectral Satellite Mission, In: Proc. IEEE Aerospace Conference, Big Sky, MT, USA, 1178.

Schneider, M., Müller, R., Krawczyk, H., Bachmann, M., Storch, T., Mogulsky, V., Hofer, S., 2012. The Future Spaceborne Hyperspectral Imager EnMAP: Its In-Flight Radiometric and Geometric Calibration Concept, In: Proc. ISPRS 2012, Melbourne, Australia, The International Archives of the Photogrammetry, Remote Sensing and Spatial Information Sciences XXXIX-B1, 267-272.

Segl, K., Guanter, L, Rogass, C., Kuester, T., Roessner, S., Kaufmann, H., Sang, B., Mogulsky, V., Hofer, S., 2012. Eetes the EnMAP end-to-end simulation tool, IEEE Journal of Selected Topics in Applied Earth Observations and Remote Sensing, 5(2), 522-530.

Storch, T., Habermeyer, M., Eberle, S., Mühle, H., Müller, R., 2013. Towards a Critical Design of an Operational Ground Segment for an Earth Observation Mission, Journal of Applied Remote Sensing, 7(1), 1-12.

Storch, T., Honold, H.-P., Krawczyk, H., Wachter, R., de los Reyes, R., Langheinrich, M., Mücke, M., Fischer, S., 2018. Spectral Characterization and Smile Correction for the Imaging Spectroscopy Mission EnMAP. In: Proc. IGARSS2018, Valencia, Spain, 3304-3306. 